\title{
Assessment of empirical formulae for determining the hydraulic conductivity of glass beads
}

\author{
Jaromír Říha, Lubomír Petrula*, Mario Hala, Zakaraya Alhasan \\ Faculty of Civil Engineering, Brno University of Technology, Veveř́ 331/95, 60200 Brno, Czech Republic. \\ ${ }^{*}$ Corresponding author. Tel.: +420 541147756. E-mail: petrula.1@fce.vutbr.cz
}

\begin{abstract}
Empirical formulae are often used in practice to quickly and cheaply determine the hydraulic conductivity of soil. Numerous relations based on dimensional analysis and experimental measurements have been published for the determination of hydraulic conductivity since the end of $19^{\text {th }}$ century. In this paper, 20 available empirical formulae are listed, converted and re-arranged into SI units. Experimental research was carried out concerning hydraulic conductivity for three glass bead size (diameters $0.2 \mathrm{~mm}, 0.5 \mathrm{~mm}$ and $1.0 \mathrm{~mm}$ ) and variable porosity. The series of experiments consisted of 177 separate tests conducted in order to obtain relevant statistical sets. The validity of various published porosity functions and empirical formulae was verified with the use of the experimental data obtained from the glass beads. The best fit was provided by the porosity function $n^{3} /(1-n)^{2}$. In the case of the estimation of the hydraulic conductivity of uniform glass beads, the best fit was exhibited by formulae published by Terzaghi, Kozeny, Carman, Zunker and Chapuis et al.
\end{abstract}

Keywords: Hydraulic conductivity; Empirical formulae; Porosity; Porosity function configuration.

\section{INTRODUCTION}

The determination of hydraulic conductivity via field pumping tests may be very costly and time-consuming. At the same time, laboratory testing using permeameters may not be a feasible solution in many cases due to time and cost restrictions. For this reason, in many practical studies, namely in preliminary aquifer assessment (EPG, 2009; Šoltész and Baroková, 2014, etc.), empirical relations appear to be a suitable alternative. However, empirical relations have been derived for specific conditions and have their applicability limits.

The typical form of empirical equations for the determination of hydraulic conductivity comes from dimensional analysis based on the Darcy-Weissbach equation (Kasenow, 2002; Vuković and Soro, 1992). The general problems with the proposed formulae lie in determining the characteristic pore diameter and expressing the effect of soil non-uniformity and the form of the appropriate porosity function which reflects the soil compaction rate.

Probably the first relation was proposed by Hazen (1892). It expresses the simple linear dependence between hydraulic conductivity and soil porosity. In his formula, Hazen did not consider the effect of soil non-uniformity. This is also the case with formulae proposed by Slichter (1899) and Terzaghi (1925). Kozeny (1927) proposed a formula that was modified by Carman $(1937,1939)$ to become the Kozeny-Carman equation.

Pavchich (VNIIG, 1991), Sauerbrey (1932), Krüger (1918), Kozeny (1953), Zunker (1932), Zamarin (1928), Koenders and Williams (1992), and Chapuis et al. (2005) derived the characteristic pore diameter from the effective grain size $d_{e}$ and porosity function $\chi(n)$ based on the analysis of typical sphere configurations (VNIIG, 1991). Most authors (Hazen, Slichter, Terzaghi, Beyer, Harleman et al., Chapuis et al., and others) considered $d_{10}$ to be an effective grain diameter, though Sauerbrey and Pavchich preferred $d_{17}$. Authors like Krüger, Kozeny, Zunker and others calculated the effective grain diameter from the grain size distribution curve. Mallet and Pacquant (1951) published frequently used tables expressing hydraulic conductivity as a function of $d_{20}$. This dependence was expressed by the United States Bureau of Reclamation (USBR) engineers via functional dependence.

Other generally less used formulae were proposed by Fair and Hatch (1933), Harleman et al. (1963), Alyamani and Sen (1993) and Chesnaux et al. (2011). The use of these formulae is restricted by their applicability limits.

The aim of the authors was to compare and assess the applicability of selected formulae. Vuković and Soro (1992) and Kasenow (2002) summarized and analysed the most important formulae with the conclusion that even when applying suitable empirical relations to the same soil sample, different resulting hydraulic conductivity values may be obtained. Further research conducted by Odong (2007) was focused on the evaluation and comparison of empirical relations with measured values. Cabalar and Akbulut (2016) measured the hydraulic conductivities of sands of different grain size and shape and compared them with some empirical formulae. Naeej et al. (2017) developed a M5 model tree used to predict hydraulic conductivity based on grain size distribution. An analysis of unconsolidated aquifer materials was performed by Hussain and Nabi (2016). Their aim was to compare seven empirical formulae with experimental data. Rosas et al. (2014) determined hydraulic conductivity from grain size distribution for 400 samples of sediments.

Some of the empirical formulae developed by different authors vaguely define applicability limits via the simple description of material type without any grain size distribution curves or quantification. This often leads to improper use of these equations. In many cases the input parameters (namely the effective grain size) in the empirical formulae need to be expressed in units other than those defined by the SI (e.g. mm, $\mathrm{cm}$ ), such as hydraulic conductivity in $\mathrm{cm} /$ day, $\mathrm{m} /$ day, etc.

The objective of this paper is to summarize the most commonly used empirical formulae, convert them strictly into SI units and evaluate their applicability and reliability for glass beads of three different diameters. The assessment of porosity functions is also included in this paper. This analytical approach enables the influence of soil non-uniformity and grain shape to be excluded from the analysis. Moreover, the grain 
size is relatively well-defined which is suggested to provide lower uncertainty in resulting hydraulic conductivities when compared with more complex soils.

First, a dimensional analysis was performed, and the dependence between porosity and pore size was established, after which the relation between hydraulic conductivity and porosity was analysed. Second, the determined empirical relations were summarized and converted into SI units. Via laboratory experiments the hydraulic conductivities of glass beads of three different diameters were determined for variable porosity. Finally, the empirical formulae were verified using the results of experimental research.

\section{DIMENSIONAL ANALYSIS}

Traditionally, the system of pores was described as the system of parallel tubes oriented in the flow direction, a conception sometimes referred to as the "Hydraulic radius model" (Bear, 1972).

The head loss $\Delta h$ is defined by the Darcy-Weissbach equation (Vuković and Soro, 1992):

$\Delta h=\lambda \frac{L}{D} \frac{v^{2}}{2 g}$,

where $L$ is the tube length, $D$ is the diameter of the tube, $v$ is the cross sectional velocity in the tube, $g$ is the gravitational acceleration, and $\lambda$ is the coefficient of friction loss, which in the case of laminar flow can be calculated as follows:

$\lambda=\frac{64}{\mathrm{Re}}$,

with the Reynolds number Re:

$\operatorname{Re}=\frac{v D}{v}$,

where $v$ is the kinematic viscosity.

The hydraulic gradient $i$ along the tube:

$i=\frac{\Delta h}{L}$

After substituting Eqs. (2 to 4) into the Darcy-Weisbach equation (1) and some manipulation, one obtains:

$i=\frac{32 v v}{D^{2} g}$.

The average velocity in pores may be expressed using the Darcy law:

$v=\frac{k i}{n_{a}}$

where $n_{a}$ is the areal porosity and $k$ is the hydraulic conductivity. Assuming areal porosity $n_{a}$ is equal to volumetric porosity $n$ (Bear, 1972), and joining Eq. (5) and (6), the hydraulic conductivity may be expressed as:

$k=\frac{1}{32} \frac{g}{v} D^{2} n$.
The tube diameter $D$ has to be substituted by the representative minimum pore diameter $d_{0}=D$ (Vuković and Soro, 1992):

$d_{0}=\alpha f(n) d_{e}$,

where $\alpha$ is a dimensionless coefficient that depends on the characteristics of the porous medium (structure, grain shape, uniformity, petrographic composition, tortuosity, etc.), $f(n)$ is the porosity function and $d_{e}$ is the effective grain diameter of the porous medium. Eq. (7) then transforms into:

$k=\frac{g}{32 v} \alpha^{2} n f^{2}(n) d_{e}^{2}$

By introducing $\chi(n)=n f^{2}(n)$ for the porosity function, Eq. (9) holds:

$k=\frac{g}{v} \beta \chi(n) d_{e}^{2}$,

where $\beta$ characterizes the properties of the porous medium and includes the constant from Eq. (8). For materials with relatively uniform grain size, such as beads, two theoretical limits to porosity may be identified (Fig. 1) according to the configuration of the grains (Indraratna and Vafai, 1997). The minimum packing corresponds to the void ratio $e_{\max }=0.908$ and maximal achievable porosity $n_{\max }=0.476$ corresponds to the ratios:

$\frac{d_{0}}{d_{\text {grain }}}=0.414, \quad \frac{d_{\max }}{d_{\text {grain }}}=0.732$,

where $d_{\text {grain }}$ is the diameter of the uniform grain and $d_{\max }$ is the maximal pore diameter.

The maximal packing gives $e_{\min }=0.351, n_{\min }=0.260$ and the ratios:

$\frac{d_{0}}{d_{\text {grain }}}=0.155, \quad \frac{d_{\max }}{d_{\text {grain }}}=0.224$

Other grain configurations are random and the resulting porosity ranges from 0.260 to 0.476 .

For non-uniform materials, Pavchich (VNIIG, 1991) proposed the following relation:

$d_{0}=0.455 \sqrt[6]{C_{U}} \frac{n}{1-n} d_{17}$

where $C_{U}$ is the coefficient of uniformity, $n$ is porosity and $d_{17}$ is the grain diameter for $17 \%$ finer by weight. In the case of a spherical grain material (like glass beads) with $C_{U} \approx 1$, Eq. (13) can be written as follows:

$\frac{d_{0}}{d_{17}}=0.455 \frac{n}{1-n}$.

\section{EMPIRICAL FORMULAE}

Empirical formulae for the hydraulic conductivity estimate $k$ stem from Eq. (10), while the porosity function is frequently determined from Eq. (13). The following list was assembled via the comparison and critical analysis of the available literature sources. All formulae have been rewritten into dimensional 


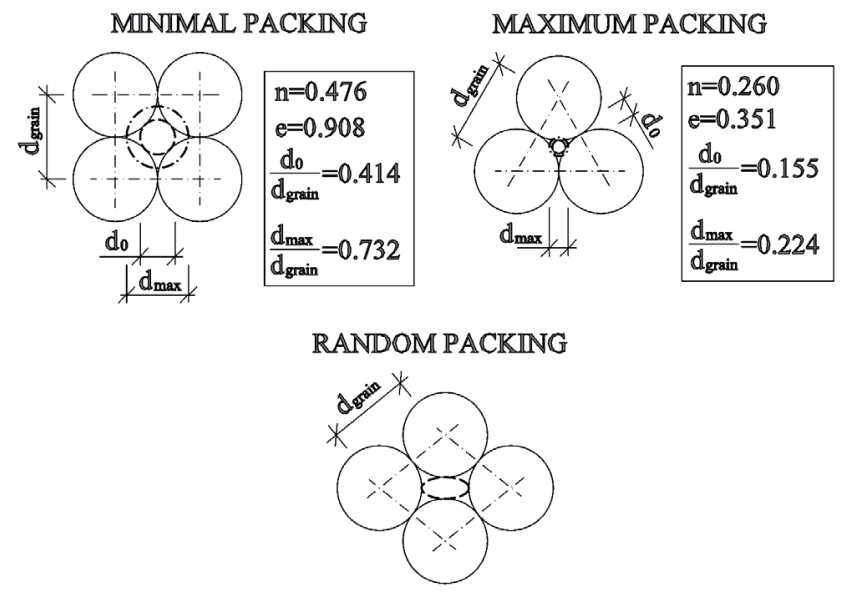

Fig. 1. Schematization of the packing of spherical grains, and possible pore size.

form (10), while the empirical coefficients have been recalculated in order to ensure SI units are used, i.e. grain diameters are expressed in [m] and hydraulic conductivity in $[\mathrm{m} / \mathrm{s}]$. The influence of temperature is included in the kinematic viscosity $\left[\mathrm{m}^{2} / \mathrm{s}\right]$.

\section{Hazen (1892)}

$k=\frac{g}{v} C_{H, 2}[1+10(n-0.26)] d_{10}^{2}$,

where $d_{10}$ is the grain diameter for $10 \%$ finer by weight and coefficient $C_{H, 2}=6 \times 10^{-4}$. Eq. (15) may be used to estimate the hydraulic conductivity of sand with $d_{\mathrm{e}}$ from 0.1 to $3 \mathrm{~mm}$ with the coefficient of uniformity $C_{U}<5$.

Slichter's (1899) formula can be used to estimate the hydraulic conductivity of soil with $d_{e}$ from 0.01 to $5.0 \mathrm{~mm}$ :

$k=\frac{g}{v} 0.01 n^{3.287} d_{10}^{2}$.

\section{Terzaghi (1925)}

$k=\frac{g}{v} C_{T}\left(\frac{n-0.13}{\sqrt[3]{1-n}}\right)^{2} d_{10}^{2}$,

where $C_{T}$ depends on the grain shape $\left(C_{T}=10.7 \times 10^{-3}\right.$ for smooth grains and $C_{T}=6.1 \times 10^{-3}$ for coarse grains). Eq. (17) may be used for large-grained sands.

\section{Beyer (1964)}

$k=\frac{g}{v} C_{B} d_{10}^{2}$,

where $C_{B}$ is:

$$
C_{B}=0.0006 \log \frac{500}{C_{U}} .
$$

This formula can be used for soils with $0.06 \leq d_{e} \leq 0.6 \mathrm{~mm}$, and with $C_{U}$ ranging from 1 to 20 .

\section{Sauerbrey (1932)}

$k=\frac{g}{v} C_{Z} \frac{n^{3}}{(1-n)^{2}} d_{17}^{2}$,

where $C_{Z}=3.75 \times 10^{-3}$. Eq. (20) can be used for soils with $d_{e}$ up to $5.0 \mathrm{~mm}$.

\section{Krüger (1918), Densch et al. (1930), Kasenow (2002)}

The Krüger (1918) formula is mentioned in several publications (Densch et al., 1930; Kasenow, 2002; Vuković and Soro, 1992) in a different form. Vuković and Soro (1992) and also Kasenow (2002) mention the following dimensional form:

$$
k=\frac{g}{v} C_{K} \frac{n}{(1-n)^{2}} d_{e}^{2},
$$

where $C_{K}=4.35 \times 10^{-3}, n$ is porosity and $d_{e}$ is effective grain defined as follows:

$$
\frac{1}{d_{e}}=\sum_{i=1}^{N} \frac{2 \Delta g_{i}}{d_{i}^{g}+d_{i}^{d}},
$$

where $\Delta g_{i}$ is the fraction of mass that passes between sieves $i$ and $i+1$ where $i$ is the smaller sieve, and $d_{i}^{g}$ and $d_{i}^{d}$ are the maximum and minimum grain diameter corresponding to the $i$ th fraction. Eqs. (21) and (22) can be used for sands of medium grain size with $C_{U}>5, N$ is the number of fractions.

However, Kasenow (2002) also mentions the form of Eq. (21) with a geometrically justified porosity function corresponding to Eqs. (20), (23), (27) and others. This form exhibits much better agreement with measured values than Eq. (21); see the Discussion section.

\section{Kozeny $(1927,1953)$}

$k=\frac{g}{v} C_{K O} \frac{n^{3}}{(1-n)^{2}} d_{e}{ }^{2}$,

where $C_{K O}=8.3 \times 10^{-3}$ and $d_{e}$ is effective grain size determined as follows:

$$
\frac{1}{d_{e}}=\frac{3 \Delta g_{1}}{2 d_{1}}+\sum_{i=2}^{N} \Delta g_{i} \frac{d_{i}^{g}+d_{i}^{d}}{2 d_{i}^{g} d_{i}^{d}}
$$

with the same notation as in Eq. (22). This formula can be used for coarse-grained sands.

\section{Zunker (1932)}

$k=\frac{g}{v} C_{Z U}\left(\frac{n}{1-n}\right)^{2} d_{e}^{2}$,

where $C_{Z U}$ is an empirical coefficient that depends on the porous medium (Table 1), $d_{e}$ is given by the formula:

$$
\frac{1}{d_{e}}=\sum_{i=1}^{N} \Delta g_{i} \frac{d_{i}^{g}-d_{i}^{d}}{d_{i}^{g} d_{i}^{d} \ln \frac{d_{i}^{g}}{d_{i}^{d}}},
$$


Table 1. Empirical coefficient for the Zunker formula (Kasenow, 2002).

\begin{tabular}{|l|c|}
\hline Characteristics of the porous medium & $C_{Z U}[-]$ \\
\hline Uniform sand with smooth, rounded grains & $2.4 \times 10^{-3}$ \\
\hline Uniform composition with coarse grains & $1.4 \times 10^{-3}$ \\
\hline Nonuniform composition & $1.2 \times 10^{-3}$ \\
\hline Nonuniform composition, clayey, with grains of irregular shape & $0.7 \times 10^{-3}$ \\
\hline
\end{tabular}

with the notation from Eq. (22). Eq. (26) can be applied for fine and medium-grained sands.

\section{Zamarin (1928)}

$k=\frac{g}{v} C_{Z A} C_{n} \frac{n^{3}}{(1-n)^{2}} d_{e}^{2}$,

where $C_{Z A}=8.64 \times 10^{-3}$ is the empirical coefficient and $C_{n}$ is a factor that depends on the porosity:

$C_{n}=(1.275-1.5 n)^{2}$.

Effective grain size $d_{e}$ is given for materials containing grains finer than $0.0025 \mathrm{~mm}$ as follows:

$\frac{1}{d_{e}}=\frac{3 \Delta g_{1}}{2 d_{1}}+\sum_{i=2}^{N} \Delta g_{i} \frac{\ln \frac{d_{i}^{g}}{d_{i}^{d}}}{d_{i}^{g}-d_{i}^{d}}$,

where $d_{1}$ is the largest diameter of the finest fraction and $\Delta g_{1}$ is the weight of the finest fraction. For materials that do not contain fractions finer than $0.0025 \mathrm{~mm}$, the effective grain size can be obtained as follows:

$\frac{1}{d_{e}}=\sum_{i=1}^{N} \Delta g_{i} \frac{\ln \frac{d_{i}^{g}}{d_{i}^{d}}}{d_{i}^{g}-d_{i}^{d}}$.

Eq. (27) can be used for fine and medium-grained sands.

\section{USBR (Mallet and Pacquant, 1951)}

$k=\frac{g}{v} C_{U S} d_{20}^{2}$,

where $d_{20}$ is the diameter of the 20 percentile grain size of the material and $C_{U S}$ is:

$C_{U S}=0.00048\left(1000 d_{20}\right)^{0.3}$.

The USBR formula, also tabulated by Mallet and Pacquant (1951), is recommended for medium-grained sands with $C_{U}<5$.

\section{Pavchich (VNIIG, 1991)}

$k=\frac{0.04}{v} \varphi_{1} \sqrt[3]{C_{U}} \frac{n^{3}}{(1-n)^{2}} d_{17}^{2}$,

where $\varphi_{1}$ is the coefficient depending on the grain size $\left(\varphi_{1}=1\right.$ for gravel sands, $\varphi_{1}=0.35-0.40$ for gravel), Eq. (33) can be used for grain sizes ranging from $0.06 \mathrm{~mm}$ to $1.5 \mathrm{~mm}$.

\section{Seelheim (1880)}

$k=3570 d_{50}^{2}$,

where $d_{50}$ is the diameter of the 50 percentile grain size. The formula was tested on sands, clay and elutriated chalk.

\section{Kozeny-Carman (Carrier, 2003)}

The following equation, which depends on the specific surface area of grains, was derived by Kozeny and Carman:

$k=\frac{g}{v} C_{K C} \frac{1}{S_{0}^{2}} \frac{n^{3}}{(1-n)^{2}}$,

where $C_{K C}=480 \pm 30$ is the empirical coefficient, and $S_{0}$ is the specific surface of particles $(1 / \mathrm{m})$. For uniform spherical grains Eq. (35) can be written as follows:

$k=\frac{g}{v} C_{K C} \frac{6}{d_{e}} d_{e}^{2} \frac{n^{3}}{(1-n)^{2}}$,

where $d_{e}$ is the uniform grain diameter $\left(d_{\text {grain }}\right)$. The formula is not appropriate for clayey soils, but it is applicable for silts, sands and gravel sands.

\section{Harleman et al. (1963)}

$k=6.54 \times 10^{-4} \frac{g}{v} d_{10}^{2}$.

\section{Koenders and Williams (1992)} tion:

This formula was derived from the Kozeny-Carman equa-

$k=\frac{1}{v} \chi n\left(\frac{n}{1-n}\right)^{2} d_{50}^{2}$,

where $\chi$ is the proportionality coefficient $(\chi=0.0035 \pm 0.0005)$ and $d_{50}$ is the median grain diameter. It is then applicable for silts, sands and gravelly sands.

The following authors used formulae that are rather different in form compared to Eq. (10).

\section{Alyamani and Sen (1993)}

$$
k=15046\left[I_{o}+0.025\left(d_{50}-d_{10}\right)\right]^{2}
$$

where $I_{o}$ is the intercept point [m] of the line formed by points $d_{50}$ and $d_{10}$ with the grain size axis. The formula can be used for well-distributed samples only.

\section{Chapuis et al. (2005)}

$$
k=1219.9 \frac{n^{2.3475}}{(1-n)^{1.565}} d_{10}^{1.565}
$$

The formula is applicable for soils with $d_{10}$ ranging from 0.03 to $3 \mathrm{~mm}$. 
Fair and Hatch (1933)

$k=\frac{g}{v} \frac{n^{3}}{(1-n)^{2}} \frac{1}{m\left(\frac{\theta}{100} \sum_{i=1}^{N} \frac{P_{i}}{d_{m i}}\right)}$,

where $m=5$ is the empirically obtained packing factor, $\theta$ is the shape factor ranging from 6 to 7.7 (spherical to angular grains), $d_{m i}$ is the geometric mean of the grain fraction, and $P_{i}$ is the percentage of sand between adjacent sieves determined by the following equation:

$P_{i}=100 w f_{i}$,

where $w_{f i}$ is the weight of the fraction retained on sieve $i$. For the geometric mean $d_{m i}$ it holds that:

$d_{m i}=\sqrt{d s_{i} d s_{i+1}}$,

where $d_{s i}$ is the size of the sieve openings for sieve $i$. This formula is applicable for sands.

NAVFAC DM7 (Chesnaux et al., 2011)

$k=0.2272\left(1.772189 \times 10^{11}\right)^{\frac{n}{1-n}}\left[d_{10}^{3.31917}\right]^{\frac{n}{1-n}}$.

The formula was derived for sands with $n$ ranging from 0.23 to $0.41, C_{U}$ ranging from 2 to $12, d_{10} / d_{5}>1.4$ and $d_{10}$ ranging from $0.1 \mathrm{~mm}$ to $2 \mathrm{~m}$

\section{EXPERIMENTAL RESEARCH}

Experiments were carried out in order to verify the porosity function and empirical formulae for uniform material laboratory experiments on glass beads of three different diameters. This experimental research aimed to obtain a sufficient number (at least 50) of hydraulic conductivity measurements for individual glass beads of different diameters with various porosities. The numbers of performed experiments are mentioned in Table 4.

\section{Equipment}

The laboratory experiments were performed using a permeameter (plastic cylinder) with upward vertical seepage flow. A permeameter consists of a cylinder containing the sample mounted on a frame. The lower part is connected to a water supply and the upper part is connected to an outlet pipe. Piezometers are located below and above the sample. The seepage flow is generated by a vertically movable water tank that can be adjusted to provide different hydraulic gradients. The movable tank is equipped with a pump that draws water from a storage tank. Water flowing through the permeameter outlet is collected and conveyed back to the storage tank. A schematic diagram of the experimental apparatus is shown in Fig. 2.

\section{Preliminary measurements}

A detailed investigation using an electron microscope showed that the sizes of the glass beads did not exactly match the interval declared by the manufacturer. Therefore, bead diameter measurements were conducted using a digital Vernier calliper for each declared (commercial) grain size in order to set up the grain size distribution curves (Fig. 3). The curves pro-

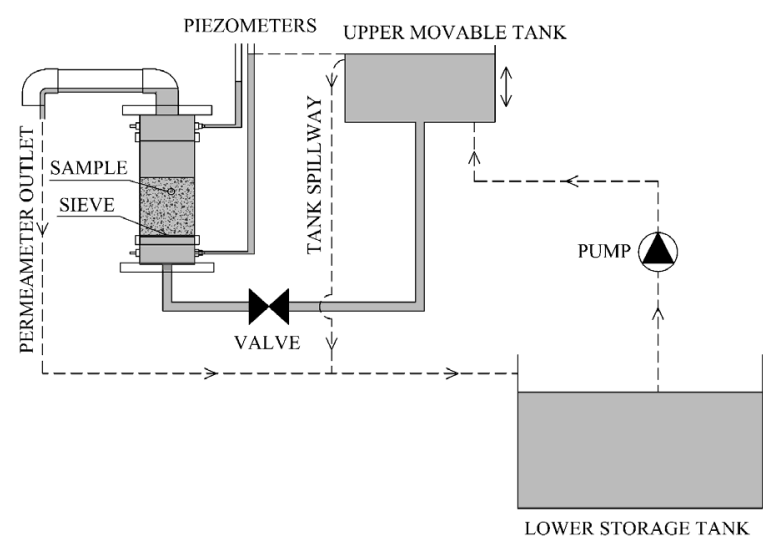

Fig. 2. Schematic diagram of the experimental apparatus.

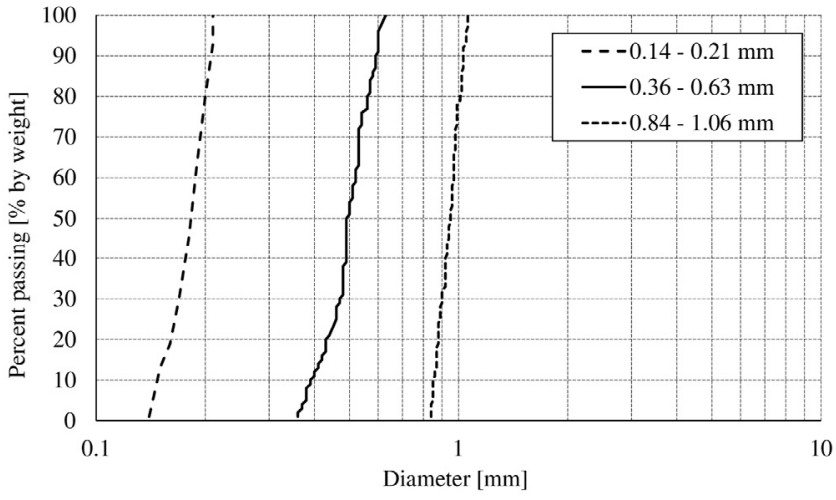

Fig. 3. Grain size distribution curves of the tested glass beads.

vided good fits with the electron microscope and the grain size characteristics were set (Table 3 ).

\section{Experimental procedure and results}

In order to obtain glass bead samples with randomly different porosity, the samples were added to the permeameter via methods involving free fall and compaction by vibration for variable durations. The porosity of each sample was determined by weighing it and then measuring its volume in a Darcy cylinder.

The various piezometric heads (and thus hydraulic gradients) were achieved by gradually raising the upper movable tank (Fig. 2). The seepage discharge was measured each time the tank was raised. In total, 177 laboratory experiments were performed on the glass beads (Table 4).

The dependence of the hydraulic conductivity $k$ on the porosity $n$ was evaluated separately for each bead diameter (Fig. 4). The porosity and hydraulic conductivity ranges are summarized in Table 4. The expected measurement accuracy for individual variables is summarised in Table 5.

\section{COMPARISON OF EXPERIMENTAL RESULTS WITH EMPIRICAL FORMULAE \\ Comparison of measured porosity with empirical results}

First, the porosity functions $\chi(n)$ used in the above-described empirical formulae (Table 2) were analysed. In Fig. 5 the correlation between porosity functions $\chi(n)$ and the ratio between measured hydraulic conductivity and effective grain size $\left(k / d_{e}{ }^{2}\right)$ is plotted. Only results with a fairly good fit are presented in Fig. 5, those being obtained by Terzaghi (1925), Sauerbrey (1932), Pavchich (VNIIG, 1991) and Chapuis et al. (2005). 
Table 2. Summary of empirical formulae.

\begin{tabular}{|c|c|c|c|c|c|}
\hline $\begin{array}{l}\text { Number of } \\
\text { formula }\end{array}$ & Author & $\beta$ & $\chi(n)$ & $d_{e}$ & Use \\
\hline 1 & Hazen (1892) & $6 \times 10^{-4}$ & {$[1+10(n-0.26)]$} & $d_{10}$ & $\begin{array}{l}\text { sands, } 0.1 \mathrm{~mm} \leq d_{10} \leq 3 \mathrm{~mm} \\
C_{U}<5\end{array}$ \\
\hline 2 & Slichter (1899) & 0.01 & $n^{3.287}$ & $d_{10}$ & $0.01 \mathrm{~mm} \leq d_{10} \leq 5 \mathrm{~mm}$ \\
\hline 3 & Terzaghi (1925) & $\begin{array}{l}10.7 \times 10^{-3}-\text { smooth } \\
\text { grains } \\
6.1 \times 10^{-3}-\text { coarse } \\
\text { grains }\end{array}$ & $\left(\frac{n-0.13}{\sqrt[3]{1-n}}\right)^{2}$ & $d_{10}$ & large-grained sands \\
\hline 4 & Beyer (1964) & $0.0006 \log \frac{500}{C_{U}}$ & & $d_{10}$ & $\begin{array}{l}0.06 \mathrm{~mm} \leq d_{10} \leq 0.6 \mathrm{~mm} \\
1 \leq C_{U} \leq 20\end{array}$ \\
\hline 5 & Sauebrej (1932) & $3.75 \times 10^{-3}$ & $\frac{n^{3}}{(1-n)^{2}}$ & $d_{17}$ & $d_{17} \leq 5 \mathrm{~mm}$ \\
\hline 6 & $\begin{array}{l}\text { Krüger (Kasenow, } \\
\text { 2002) }\end{array}$ & $4.35 \times 10^{-3}$ & $\frac{n}{(1-n)^{2}}$ & Eq. (22) & $\begin{array}{l}\text { sands of medium grain size } \\
C_{U}>5\end{array}$ \\
\hline 7 & Kozeny (1953) & $8.3 \times 10^{-3}$ & $\frac{n^{3}}{(1-n)^{2}}$ & Eq. (24) & coarse-grained sands \\
\hline 8 & Zunker (1932) & Table 1 & $\left(\frac{n}{1-n}\right)^{2}$ & Eq. (26) & $\begin{array}{l}\text { fine and medium-grained } \\
\text { sands }\end{array}$ \\
\hline 9 & Zamarin (1928) & $8.64 \times 10^{-3}$ & $\frac{n^{3}}{(1-n)^{2}}(1.275-1.5 n)^{2}$ & $\begin{array}{l}\text { Eq. (29) } \\
\text { Eq. (30) }\end{array}$ & $\begin{array}{l}\text { fine and medium-grained } \\
\text { sands }\end{array}$ \\
\hline 10 & $\begin{array}{l}\text { USBR (Mallet and } \\
\text { Pacquant, 1951) }\end{array}$ & $0.00048\left(1000 d_{20}\right)^{0.3}$ & & $d_{20}$ & $\begin{array}{l}\text { medium-grained sands } \\
C_{U}<5 ; T=15^{\circ} \mathrm{C}\end{array}$ \\
\hline 11 & $\begin{array}{l}\text { Pavchich } \\
\text { (VNIIG, 1991) }\end{array}$ & $0.04 \varphi_{1} \sqrt[3]{C_{U}}$ & $\frac{n^{3}}{(1-n)^{2}}$ & $d_{17}$ & $0.06 \mathrm{~mm} \leq d_{17} \leq 1.5 \mathrm{~mm}$ \\
\hline 12 & Seelheim (1880) & 3570 & & $d_{50}$ & $\begin{array}{l}\text { sands, clay and elutriated } \\
\text { chalk }\end{array}$ \\
\hline 13 & $\begin{array}{l}\text { Kozeny-Carman } \\
\text { (Carrier, 2003) }\end{array}$ & $480 \pm 30$ & $\frac{n^{3}}{(1-n)^{2}}$ & $d_{\text {grain }}$ & uniform spherical grains \\
\hline 14 & $\begin{array}{l}\text { Harleman et al. } \\
(1963)\end{array}$ & $6.54 \times 10^{-4}$ & & $d_{10}$ & \\
\hline 15 & $\begin{array}{l}\text { Koenders and } \\
\text { Williams (1992) }\end{array}$ & $0.0035 \pm 0.0005$ & $n\left(\frac{n}{1-n}\right)^{2}$ & $d_{50}$ & $\begin{array}{l}\text { silts, sands and gravelly } \\
\text { sands }\end{array}$ \\
\hline 16 & $\begin{array}{l}\text { Alyamani and Sen } \\
(1993)\end{array}$ & 15046 & & $I_{O}, d_{50}, d_{10}$ & well-distributed sample \\
\hline 17 & $\begin{array}{l}\text { Chapuis et al. } \\
\text { (2005) }\end{array}$ & 1219.9 & $\frac{n^{2.3475}}{(1-n)^{1.565}}$ & $d_{10}$ & $0.03 \mathrm{~mm} \leq d_{10} \leq 3 \mathrm{~mm}$ \\
\hline 18 & $\begin{array}{l}\text { Fair and Hatch } \\
\text { (1933) }\end{array}$ & 1 & $\frac{n^{3}}{(1-n)^{2}}$ & $m\left(\frac{\theta}{100} \sum_{i=1}^{N} \frac{P_{i}}{d_{m i}}\right)$ & $\begin{array}{l}\text { sands } \\
m=5 \\
6 \leq \theta \leq 7.7\end{array}$ \\
\hline 19 & $\begin{array}{l}\text { NAVFAC DM7 } \\
\text { (Chesnaux et al., } \\
\text { 2011) }\end{array}$ & 0.2272 & $\left(1.772189 \cdot 10^{11}\right)^{\frac{n}{1-n}}$ & $d_{10}$ & $\begin{array}{l}0.23 \leq n \leq 0.41 \\
2 \leq C_{U} \leq 12 \\
d_{10} / d_{5}>1.4 \\
0.1 \mathrm{~mm} \leq d_{10} \leq 2 \mathrm{~mm}\end{array}$ \\
\hline
\end{tabular}

Table 3. Grain size characteristics.

\begin{tabular}{|c|c|c|c|c|c|c|c|}
\hline \multirow{2}{*}{$\begin{array}{c}\text { Diameter } \\
d_{\min }-d_{\max }\end{array}$} & $d_{10}$ & $d_{17}$ & $d_{20}$ & $d_{60}$ & $d_{e, \text { Krïger, Zamarin, Zunker }}$ & $d_{e, \text { Kozeny }}$ & $C_{U}$ \\
\cline { 2 - 8 } & $\mathrm{mm}$ & $\mathrm{mm}$ & $\mathrm{mm}$ & $\mathrm{mm}$ & $\mathrm{mm}$ & $\mathrm{mm}$ & - \\
\hline $0.14-0.21$ & 0.16 & 0.17 & 0.17 & 0.19 & 0.18 & 0.18 & 1.19 \\
\hline $0.36-0.63$ & 0.39 & 0.43 & 0.43 & 0.52 & 0.49 & 0.48 & 1.33 \\
\hline $0.84-1.06$ & 0.85 & 0.87 & 0.88 & 0.97 & 0.94 & 0.92 & 1.14 \\
\hline
\end{tabular}

For each data set a linear relation between the hydraulic conductivity and the porosity function was assumed depending on
Eq. (10). Determination coefficients were evaluated in order to assess the best fit. 
Table 4. Summary of performed experiments and the minimum and maximum values of porosity and hydraulic conductivity.

\begin{tabular}{|c|c|c|c|c|c|}
\hline \multirow{2}{*}{ Grain diameter } & \multirow{2}{*}{$\begin{array}{c}\text { Number of } \\
\text { experiments }\end{array}$} & \multicolumn{2}{|c|}{ Porosity } & \multicolumn{2}{c|}{ Hydraulic conductivity } \\
\cline { 3 - 5 } & {$[-]$} & Minimum & Maximum & Minimum & Maximum \\
\hline$[\mathrm{mm}]$ & 52 & 0.377 & {$[-]$} & {$[\mathrm{m} / \mathrm{s}]$} & {$[\mathrm{m} / \mathrm{s}]$} \\
\hline $0.14-0.21$ & 53 & 0.368 & 0.446 & 0.00416 & 0.00922 \\
\hline $0.36-0.63$ & 72 & 0.353 & 0.416 & 0.00110 & 0.00220 \\
\hline $0.84-1.06$ & & & 0.00013 & 0.00036 \\
\hline
\end{tabular}

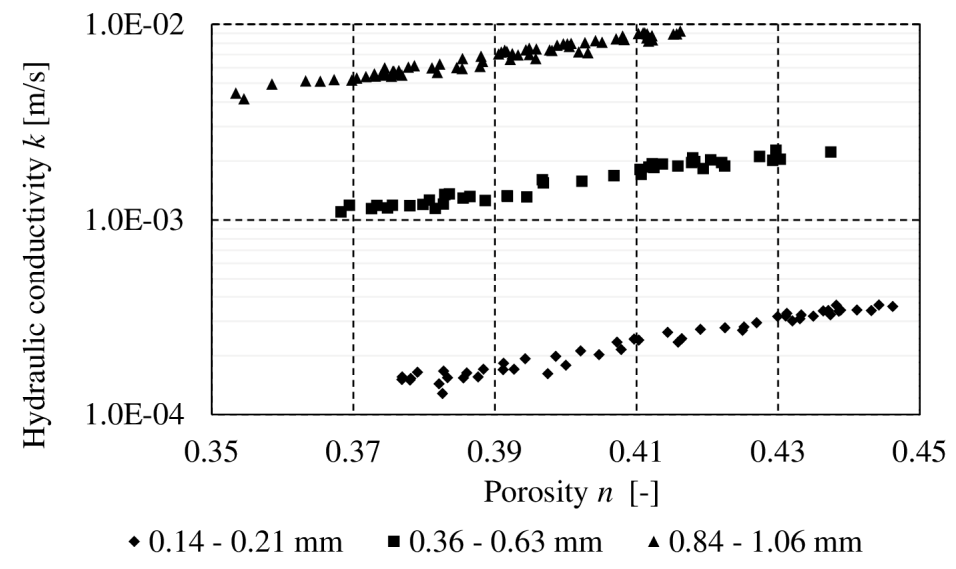

Fig. 4. Dependence of hydraulic conductivity on porosity.

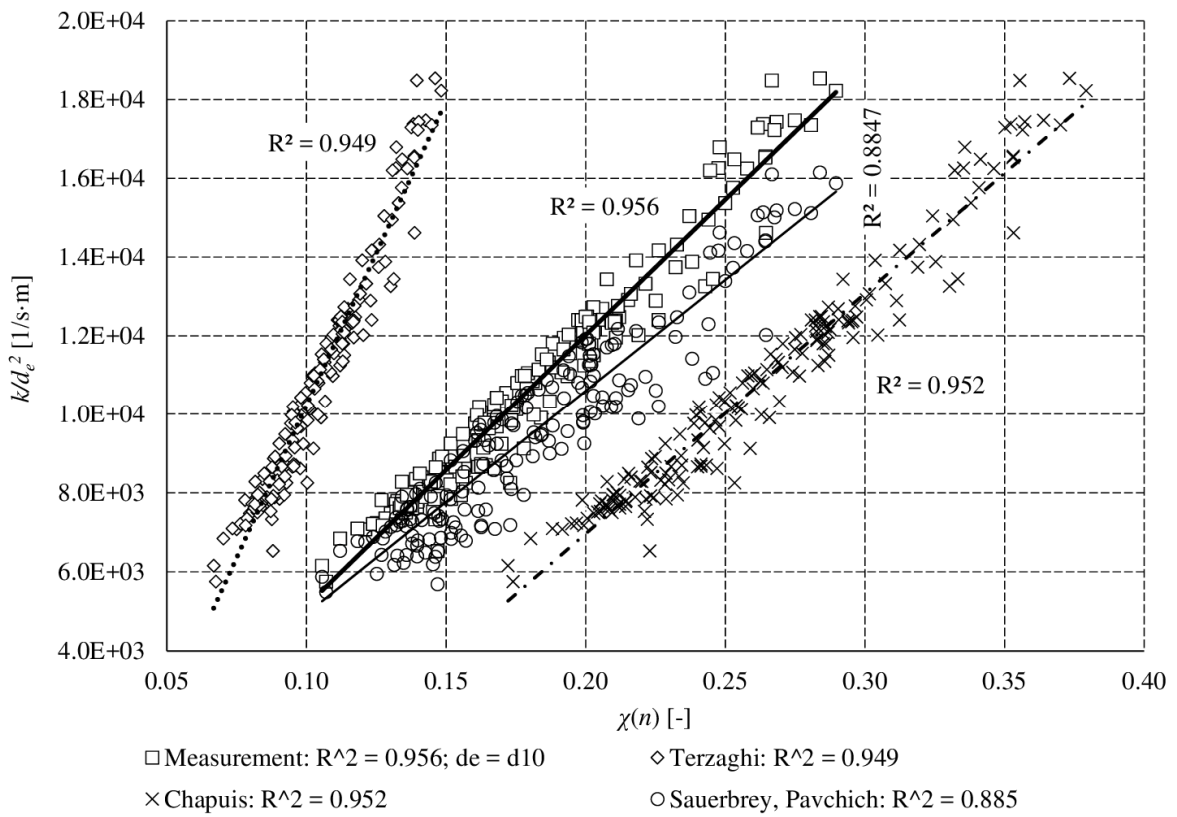

Fig. 5. Correlation between $\chi(n)$ and $\left(k / d_{e}^{2}\right)$.

Table 5. Summary of measurement accuracy.

\begin{tabular}{|l|c|}
\hline \multicolumn{2}{|c|}{ Accuracy of directly measured variables } \\
\hline \multicolumn{1}{|c|}{ Variable } & Accuracy \\
\hline Glass bead diameter & $0.01 \mathrm{~mm}$ \\
\hline Weight & $0.0001 \mathrm{~kg}$ \\
\hline Permeameter diameter & $0.25 \mathrm{~mm}$ \\
\hline Length of sample & $025 \mathrm{~mm}$ \\
\hline Piezometric heights & $0.25 \mathrm{~mm}$ \\
\hline Temperature & $0.25^{\circ} \mathrm{C}$ \\
\hline Time & $0.05 \mathrm{~s}$ \\
\hline
\end{tabular}

The dependence between the most frequently used porosity function from Eq. (10) and the measured hydraulic conductivities and for $d_{e}=d_{10}$ was added to the graph. This provided the best fit with $\mathrm{R}^{2} \approx 0.956$. As regards the empirical formulae, the closest values to the measured data were obtained from the Chapuis (2005) and empirical Terzaghi (1925) porosity functions. A relatively good fit was also provided by the geometrically based relation (14) derived by Pavchich (VNIIG, 1991) and Sauerbrey (1932) with $\mathrm{R}^{2} \approx 0.885$. 


\section{Comparison of the measured hydraulic conductivity with the empirical formulae}

Figs. 6 to 10 show comparisons of the measured conductivity values with the calculated values gained from the empirical formulas. The ratio $k / d_{e}^{2}$ used in the plots enables the joint comparison of results for all tested bead diameters. This comparison was not performed for formulae that do not meet applicability limits, such as Alyamani and Sen (1993), Fair and Hatch (1933) and Chesnaux et al. (2011).

To quantify the rate of agreement numerically, the sums of the standardised squares of the residuals $\Sigma \varepsilon$ were expressed in Table 6:

$\Sigma \varepsilon=\sum_{i=1}^{N} \frac{\left(k_{i-\text { calculated }}-k_{i-\text { measured }}\right)^{2}}{k_{i-\text { measured }}^{2}}$

where $k_{i-c a l c u l a t e d}$ and $k_{i-m e a s u r e d}$ are hydraulic conductivities obtained from empirical formulae and from measurements, respectively, and $N=177$ is the number of measurements.

\section{DISCUSSION}

The analysis of the porosity functions shows that the best fit is provided by a commonly used porosity function based on Eq. (13), or on (14) with the effective grain $d_{10}$. A quite good fit is also achieved by the dependence proposed by Terzaghi (1925). This is especially true for the measured hydraulic conductivities when $d_{e}=d_{17}$.

In Figs. 6 to 10 it can be seen that there are considerable differences between the empirical formulae listed above. This is because the individual formulae were derived for specific conditions via different methods. Some are geometrically and physically justified, while others are pure regression dependencies which are not supported by dimensional analysis.

A visual check of Figs. 6 to 10 indicates that for uniform glass beads the best fit with the measured hydraulic conductivities is provided by the formulae published by Terzaghi (Fig. 6), Kozeny-Carman, Zunker (Fig. 8) and Chapuis et al. (Fig. 9). A still reasonable degree of agreement is given by the formulae by Hazen, Zamarin, Sauerbrey and Pavchich.

Table 6. Sums of standardized squared deviations for empirical formulae (ascending order).

\begin{tabular}{|l|c|c|c|}
\hline Author & Formula number in Table 2 & Eq. number & Sum of standardized squared deviations $\Sigma \varepsilon$ \\
\hline Kozeny-Carman (Carrier, 2003) & 13 & $(35)$ & 1.25 \\
\hline Zunker (1932) & 8 & $(25)$ & 1.38 \\
\hline Terzaghi (1925) & 3 & $(17)$ & 1.80 \\
\hline Zamarin (1928) & 9 & $(27)$ & 7.07 \\
\hline Pavchich (VNIIG, 1991) & 11 & $(33)$ & 7.45 \\
\hline Sauerbrey (1932) & 5 & $(20)$ & 16.36 \\
\hline USBR (Mallet and Pacquant, 1951) & 10 & $(31)$ & 18.80 \\
\hline Chapuis et al. (2005) & 17 & $(40)$ & 26.47 \\
\hline Kozeny (1953) & 7 & $(23)$ & 28.25 \\
\hline Harleman et al. (1963) & 14 & $(37)$ & 30.91 \\
\hline Beyer (1964) & 4 & $(18)$ & 31.60 \\
\hline Hazen (1892) & 1 & $(15)$ & 46.34 \\
\hline Seelheim (1880) & 12 & $(34)$ & 51.29 \\
\hline Slichter (1899) & 2 & $(16)$ & 145.47 \\
\hline Koenders and Williams (1992) & 15 & $(38)$ & 5284.86 \\
\hline Krüger (Kasenow, 2002) & 6 & & \\
\hline
\end{tabular}

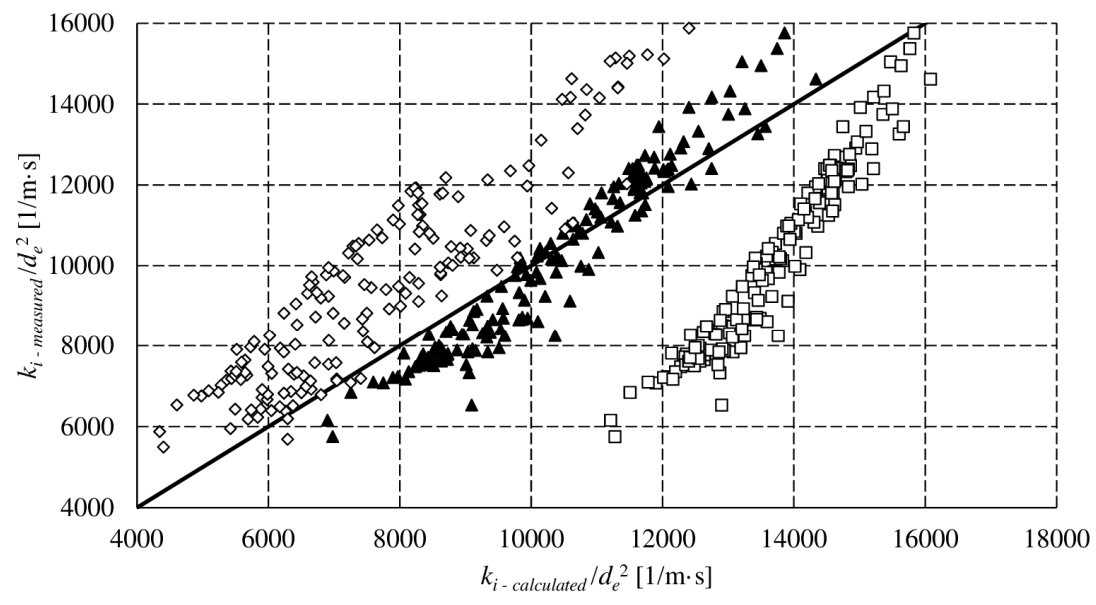

- Agreement line $\quad \square$ Hazen (1892) $\Delta$ Terzaghi (1925) $\diamond$ Pavchich (VNIIG, 1991)

Fig. 6. Comparison of calculated and measured hydraulic conductivity - part 1 . 


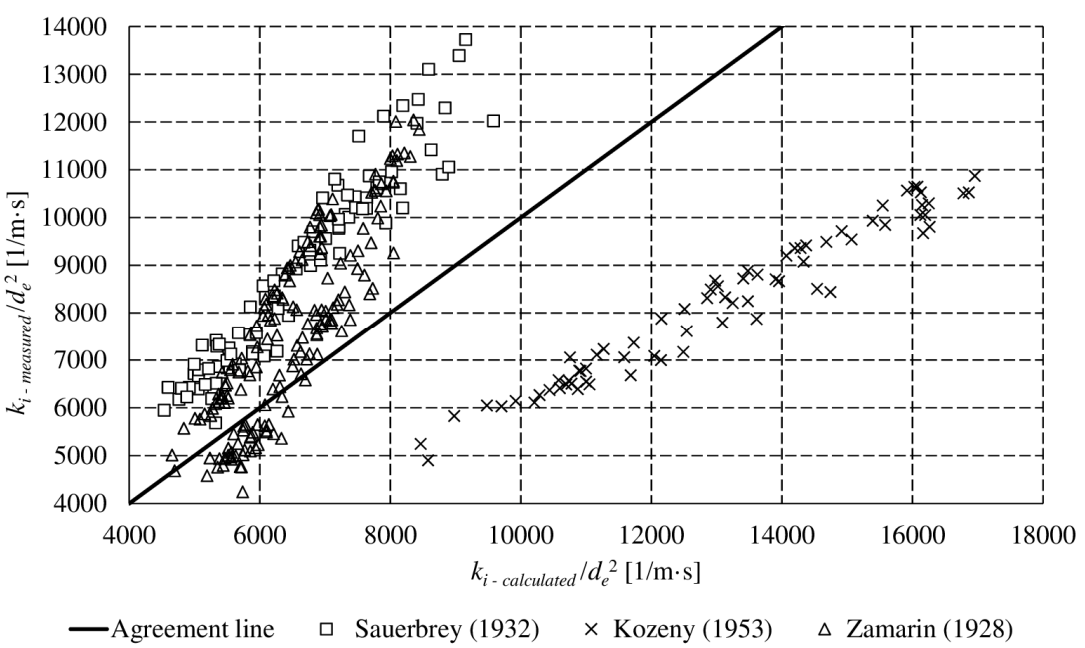

Fig. 7. Comparison of calculated and measured hydraulic conductivity - part 2.

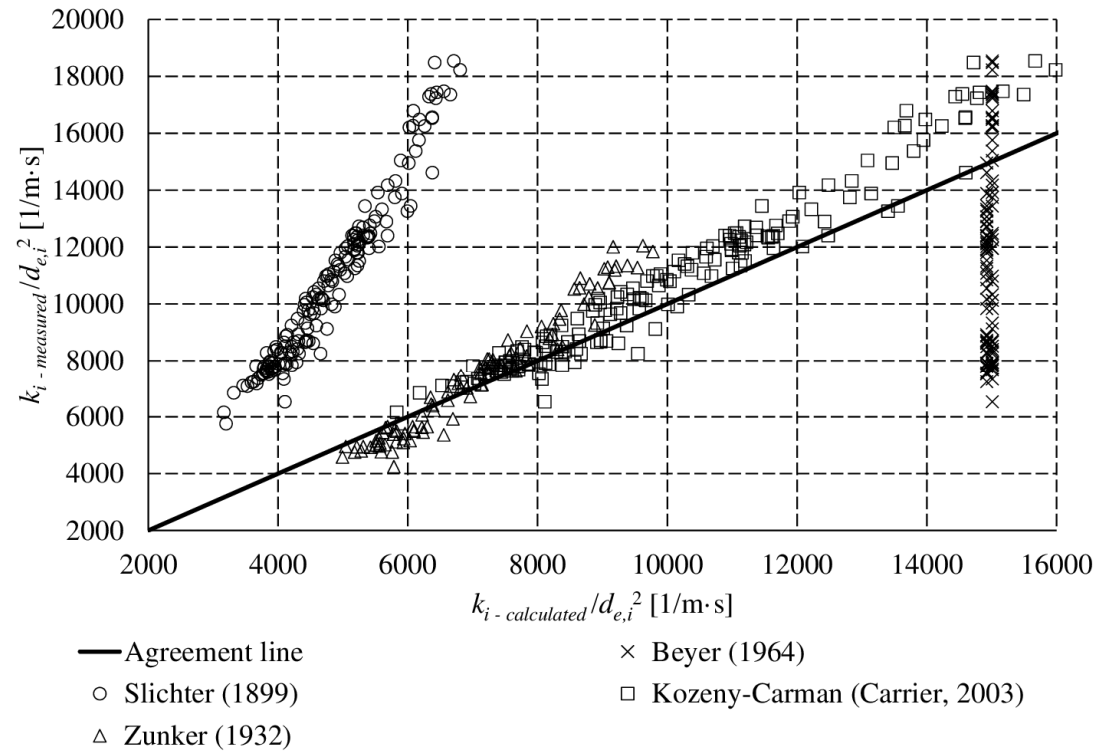

Fig. 8. Comparison of calculated and measured hydraulic conductivity - part 3.

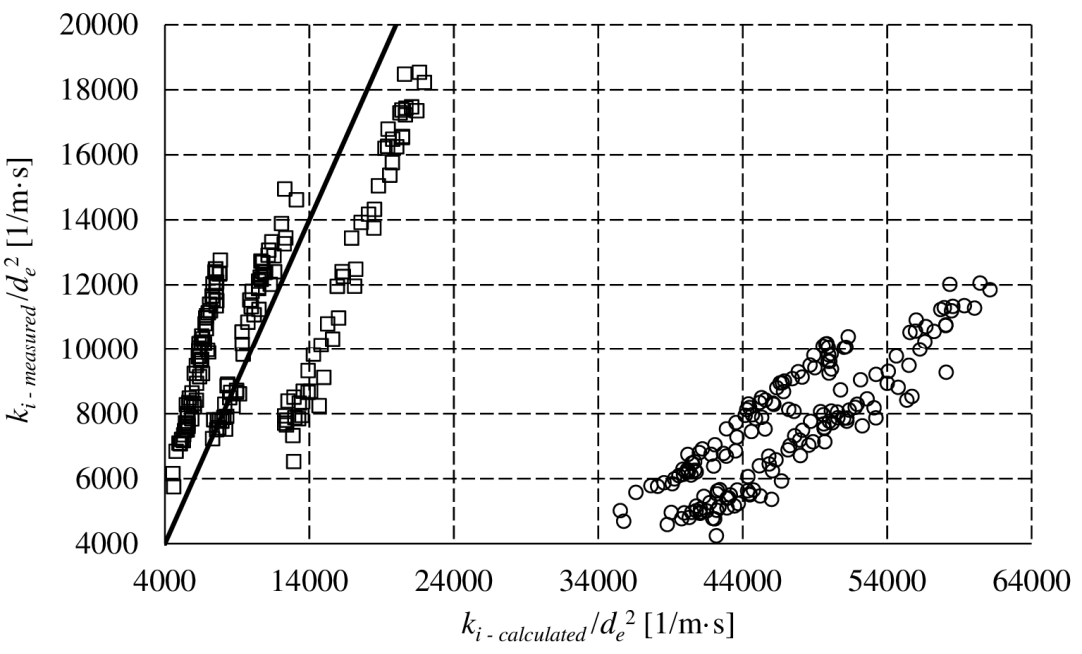

—Agreement line $\quad \circ$ Krüger (Kasenow, 2002) $\square$ Chapuis et al. (2005)

Fig. 9. Comparison of calculated and measured hydraulic conductivity - part 4 . 


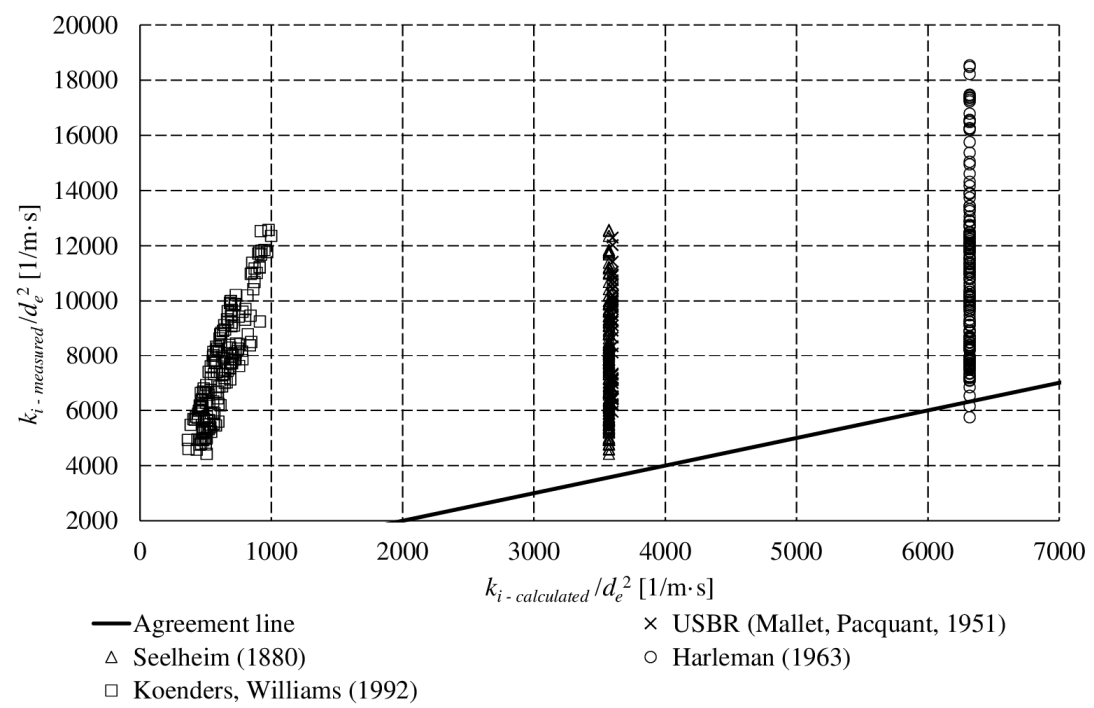

Fig. 10. Comparison of calculated and measured hydraulic conductivity - part 5.

Table 7. - Ratios of calculated and measured values.

\begin{tabular}{|c|c|c|c|}
\hline \multirow{2}{*}{$\begin{array}{c}\text { Formula number } \\
\text { in Table 2 }\end{array}$} & \multirow{2}{*}{$\begin{array}{c}\text { Equation } \\
\text { number }\end{array}$} & \multicolumn{2}{|c|}{ Agreement ratio } \\
\cline { 3 - 4 } & $(15)$ & 0.87 & 1.98 \\
\hline 1 & $(16)$ & 0.35 & 0.63 \\
\hline 2 & $(17)$ & 0.78 & 1.39 \\
\hline 3 & $(18)$ & 0.81 & 2.30 \\
\hline 4 & $(20)$ & 0.60 & 0.94 \\
\hline 5 & $(21)$ & 4.85 & 9.93 \\
\hline 6 & $(23)$ & 1.49 & 1.75 \\
\hline 7 & $(25)$ & 0.76 & 1.36 \\
\hline 8 & $(27)$ & 0.67 & 1.35 \\
\hline 9 & $(31)$ & 0.29 & 0.60 \\
\hline 10 & $(33)$ & 0.68 & 1.11 \\
\hline 11 & $(34)$ & 0.28 & 0.81 \\
\hline 12 & $(35)$ & 0.80 & 1.24 \\
\hline 13 & $(37)$ & 0.34 & 1.10 \\
\hline 14 & $(38)$ & 0.07 & 0.11 \\
\hline 15 & $(40)$ & 0.60 & 1.98 \\
\hline 17 & & & \\
\hline & & & \\
\hline & & &
\end{tabular}

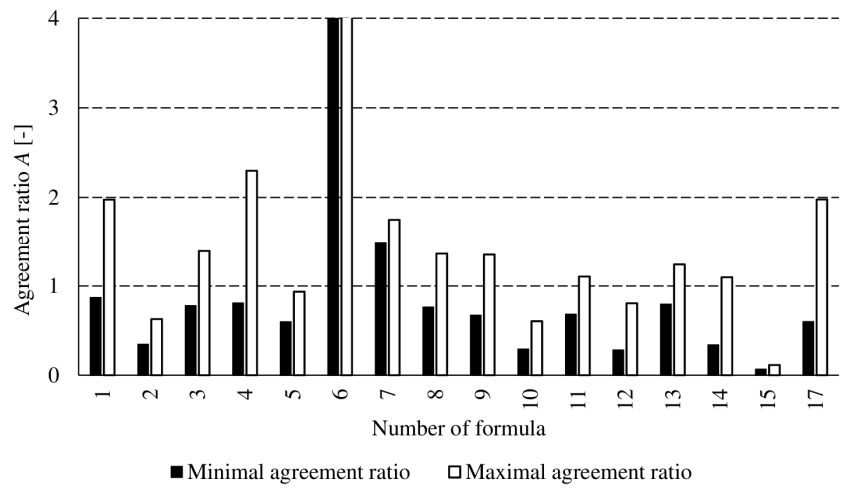

Fig. 11. Minimal and maximal agreement ratios.

In contrast, the worst agreement is provided by the formula derived by Krüger and also formulae which do not take the porosity effect into account, specifically those by Seelheim, USBR, Harleman et al. and Beyer, which show practically no agreement. Interesting results were achieved when the porosity function $n^{3} /(1-n)^{2}$ was implemented into the Krüger formula.
The originally poor fit shown in Fig. 9 was significantly improved by this alteration. Other empirical formulae tend to overestimate or underestimate the hydraulic conductivity more significantly in comparison to conducted measurements. To gain an idea about the rate of agreement, the ratios between the calculated and experimentally measured hydraulic conductivity values were computed:

$A=\frac{k_{\text {emp }}}{k_{\text {mea }}}, A_{\min }=\min (A), A_{\max }=\max (A)$

where $A$ is the agreement ratio, $k_{\text {emp }}$ is the hydraulic conductivity obtained from an empirical formula, and $k_{\text {mea }}$ is the hydraulic conductivity obtained from measurements. The ratio $A$ was enumerated for all measurements and formulae and its minimum and maximum values were identified for each formula (Table 7, Fig. 11). The best fit is represented by $A=1.0$.

From the graphs in Figs. 6, 7, 9 two or three slightly different clusters in the term $k / d_{e}^{2}$ may be identified for individual formulae, namely e.g. Hazen (Fig. 6), Zamarin (Fig. 7), Krüger and Chapuis (Fig. 9). This fact may be attributed to the increasing effect of surface tension with decreasing grain size, a factor which is not included in the porosity function. Here, the "effective porosity" (Bear, 1972) should be used instead of "dry" porosity in empirical equations.

\section{CONCLUSION}

In the study, empirical formulae for determining hydraulic conductivity were presented and transformed into dimensional form using SI units ( $\mathrm{m}, \mathrm{m} / \mathrm{s}$, etc.). The advisability of using porosity functions in empirical formulae was examined along with their applicability for uniform spherical grains using the results of 177 laboratory tests on glass beads of three different diameters.

The best fit was provided by the geometrically derived porosity function $n^{3} /(1-n)^{2}$ based on Eq. (13) when $d_{\mathrm{e}}=d_{10}$ was used. For uniform glass beads the best fit was exhibited by formulae published by Terzaghi (Eq. (17)), Kozeny-Carman (Eq. (36)), Zunker (Eqs. $(25,26))$ and Chapuis et al. (Eq. (40)). If applied porosity function $n^{3} /(1-n)^{2}$ into the Krüger formula Eq. (21) very good fit with measured values is also achieved. 
The comparison shows the increasing effect of surface tension and capillary forces with decreasing grain size. Further research should be focused on this effect and the use of "effective porosity" instead of standard porosity in empirical formulae.

Acknowledgement. This study was conducted as part of the following projects: No. LO1408 AdMaS UP - Advanced Materials, Structures and Technologies; FAST-S-17-4066 The assessment of the filtration instability origin in the soils by the limit state method; and FAST-J-17-4688: Laboratory measurement of internal erosion.

\section{REFERENCES}

Alyamani, M.S., Sen, Z., 1993. Determination of hydraulic conductivity from complete grain-size distribution. Ground Water, 31, 4, 551-555.

Bear, J., 1972. Dynamics of Fluids in Porous Media. Elsevier Publishing Company, Amsterdam, 764 p.

Beyer, W., 1964. On the determination of hydraulic conductivity of gravels and sands from grain-size distribution. Wasserwirtschaft Wassertechnik, 14, 165-169. (In German.)

Cabalar, A.F., Akbulut, N., 2016. Evaluation of actual and estimated hydraulic conductivity of sands with different gradation and shape. SpringerPlus, 5, 1, 820.

Carman, P.C., 1937. Fluid flow through granular beds. Transactions, Institution of Chemical Engineers, London, 15, 150166.

Carman, P.C., 1939. Permeability of saturated sands, soils and clays. Journal of Agricultural Science, 29, 263-273.

Carrier, W.D., 2003. Goodbye, Hazen; Hello, Kozeny-Carman. Journal of Geotechnical and Geoenvironmental Engineering, 129, 11, 1054-1056.

Chapuis, R.P., Dallaire, V., Marcotte, D., Chouteau, M., Acevedo, N., Gagnon, F., 2005. Evaluating the hydraulic conductivity at three different scales within an unconfined sand aquifer at Lachenaie, Quebec. Canadian Geotechnical Journal, 42, 1212-1220.

Chesnaux, R., Baudement, C., Hay, M., 2011. Assessing and comparing the hydraulic properties of granular aquifers on three different scales. In: Proceedings of Geohydro 2011, Quebec City, QC, Canada, 9 p.

Densch, A., Giesecke, N., Helbig, N., Heß, N., Schubert, N., Zunker, N., 1930. Die Physikalische Beschaffenheit des Bodens. Springer Verlag, Berlin. 424 p.

EPG, 2009. The sustainable management of groundwater in Canada. Report of the Expert Panel on Groundwater. ISBN 978-1-926558-11-0. Government of Canada, Ottawa, 254 p.

Fair, G.M., Hatch, L.P., 1933. Fundamental factors governing the streamline flow of water through sand. J. Amer. Water Works Assoc., 25, 1551-1565.

Harleman, D.R.F., Melhorn, P.F., Rumer, R.R., 1963. Dispersion-permeability correlation in porous media. Journal of the Hydraulic Division, 89, 2, 67-85.

Hazen, A., 1892. Some physical properties of sand and gravel with special reference to their use in filtration. Rept. Massachusetts. State Board of Health, Boston.

Hussain, F., Nabi, G., 2016. Empirical formulae evaluation for hydraulic conductivity determination based on grain size analysis. Int. J. Res. Env. Std., 3, 3, 26-32.
Indraratna, B., Vafai, F., 1997. Analytical model for particle migration within base soil-filter system. J. Geotech. and Geoenviron. Eng., ASCE, 123, 2, 100-109.

Kasenow, M., 2002. Determination of Hydraulic Conductivity from Grain Size Analysis. Water Resources Publications, LLC, Highland Ranch, CO, USA, 83 .

Koenders, M.A., Williams, A.F., 1992. Flow equations of particle fluid mixtures. Acta Mechanica, 92, 91-116.

Kozeny, J., 1927. Über kapillare Leitung der Wasser in Boden. Royal Academy of Science, Vienna, Proc. Class I, 136, pp. 271-306.

Kozeny, J., 1953. Das Wasser in Boden, Grundwasserbewegung. In: Kozeny, J. Hydraulik. Springer, Vienna, pp. 380-445. (In German.)

Krüger, E., 1918. Die Grundwasserbewegung. Internat. Mitt. Bodenkunde. Band VIII, Heft 5/6, pp. 105-122.

Mallet, C., Pacquant, J., 1951. Les barrages en terre. Editions Eyrolles, Paris, 345 p.

Naeej, M., Naeej, M.R., Salehi, J., Rahimi, R., 2017. Hydraulic conductivity prediction based on grain-size distribution using M5 model tree. Geomechanics and Geoengineering, 12, $2,107-114$

Odong, J., 2007. Evaluation of empirical formulae for determination of hydraulic conductivity based on grain-size analysis. The Journal of American Science, 3, 3, 54-60.

Rosas, J., Lopez, O., Missimer, T.M., Coulibaly, K.M., Dehwah, A.H.A., Sesler, K., Lujan, L.R., Mantilla, D., 2014. Determination of hydraulic conductivity from grain-size distribution for different depositional environments. Groundwater, 52, 3, 399-413.

Sauerbrey, I.I., 1932. On the Problem and Determination of the Permeability Coefficient. Proceedings VNIIG, No. 3-5. (In Russian.)

Seelheim, F., 1880. Methoden zur Bestimmung der Durchlassigkeit des Bodens. Analytical and Bioanalytical Chemistry, 19, 1, 387-418.

Slichter, C.S., 1899. Theoretical investigation of the motion of ground waters. U.S. Geological Survey 19th Annual Report, Part 2. United States Government Printing Office, Washington, DC, USA, $322 \mathrm{p}$.

Šoltész, A., Baroková, D., 2014. Analysis of surface and ground water interaction in the Danube river branch system. In: 14th International Multidisciplinary Scientific GeoConference SGEM 2014, Bulgaria, Vol. 1, Issue 3, 2014, pp. 5158.

Terzaghi, C., 1925. Principles of Soil Mechanics. Engineering News Record, 95, 832 p.

VNIIG, 1991. Recommendations on the Laboratory Methods of Investigation of the Permeability and Filtration Stability of Soils. P 49-90/VNIIG. The B. E. Vedeneev All-Russia Research Institute of Hydraulic Engineering, JSC, Leningrad, 93 p. (In Russian.)

Vuković, M., Soro, A., 1992. Determination of Hydraulic Conductivity of Porous Media from Grain-Size Composition. Water Resources Publications, Littleton, CO, USA, 87 p.

Zamarin, E.A., 1928. The Calculus of the Groundwater Flow. Izd-vo IVCH, Tashkent. (In Russian.)

Zunker, F., 1932. Fertilization and soil science. Journal of Plant Nutrition, A25. (In German.)

Received 20 December 2017 Accepted 12 April 2018 